\title{
Abnormal Lipid Composition of Chylomicrons in Broad- $\beta$ Disease (Type III Hyperlipoproteinemia)
}

\author{
Wirliam R. Hazzard, Daniel Porte, Jr., and Edwin L. Bierman \\ From the Department of Medicine, University of Washington School of \\ Medicine and VA Hospital, Seattle, Washington 98108
}

A B S T R A C T Chylomicron (primary particles) were detected by polyvinylpyrollidone (PVP) flocculation in plasma collected after an overnight fast from eight hyperlipemic subjects with broad- $\beta$ disease (type III hyperlipoproteinemia). The composition of these chylomicrons was abnormal: relatively poor in triglyceride and rich in cholesterol, giving rise to a triglyceride/ cholesterol ratio of $<3.0$ in all cases, uniformly below the ratio in chylomicrons from eight fasting subjects with mixed lipemia. By contrast, at the peak of alimentary lipemia following an oral fat load $(2 \mathrm{~g} / \mathrm{kg})$, chylomicrons from broad- $\beta$ subjects had normal, triglyceride-rich composition (triglyceride/cholesterol = 14.0) and resembled chylomicrons from subjects with mixed lipemia, endogenous lipemia, and familial hypercholesterolemia after similar fat loads. As the alimentary lipemia cleared, chylomicrons remaining in broad- $\beta$ subjects 14-24 hr after the fat load were again rich in cholesterol. However, a similar degree of cholesterol enrichment was observed in chylomicrons from the subjects with familial hypercholesterolemia, while only a minor increase in cholesterol was recorded in chylomicrons from subjects with mixed or endogenous lipemia. Parallel studies of changes in chylomicron composition during in vitro incubation of whole plasma and of $S_{\mathfrak{t}}>$ 400 with $S_{e}<400$ lipoproteins from subjects with the different forms of hyperlipoproteinemia revealed equal cholesterol enrichment of chylomicrons from a subject with mixed lipemia and from a subject with broad- $\beta$ disease in media of equivalent cholesterol content. These experiments suggested neither excessive avidity of chylomicrons for cholesterol uptake nor excessive influence of $\mathrm{S}_{\mathrm{t}}<400$ lipoproteins upon chylomicron composition in broad- $\beta$ disease.

Thus, results in this study suggest that the cholesterolrich chylomicrons observed in subjects with broad- $\beta$ disease after an overnight fast may originate in the in-

This work was presented in part at the Western Society for Clinical Research, Carmel, Calif., January 1969.

Received for publication 16 April 1970 and in revised form 1 June 1970. testine as particles of normal composition (chiefly dietary triglyceride) but assume a composition which is relatively rich in cholesterol through processes of lipolysis and cholesterol transfer among circulating lipoproteins which may not be unique to broad- $\beta$ disease.

\section{INTRODUCTION}

Broad- $\beta$ disease (type III hyperlipoproteinemia) (1) is associated with premature atherosclerosis, planar and tuberous xanthomas, and both hypercholesterolemia and hypertriglyceridemia. Very low density lipoproteins (VLDL) $\left(\mathrm{d}<1.006, \mathrm{~S}_{\mathrm{t}}>20\right)$ in this disorder are distinctive in at least two respects: (a) a portion migrate with $\beta$-lipoproteins to produce a "broad- $\beta$ " pattern upon electrophoresis of whole plasma (1), and (b), they are relatively rich in cholesterol and poor in triglyceride (TG) $(1,2)$. In the present study chylomicrons ${ }^{1}$ were found to persist after an overnight fast in lipemic subjects with broad- $\beta$ disease and to reflect the cholesterolrich nature of VLDL as a whole. The mechanism of this compositional abnormality has been explored both in vivo and in vitro in studies of chylomicron lipid content during the assimilation of dietary fat in subjects with broad- $\beta$ disease.

\section{METHODS}

Subjects. All subjects with broad- $\beta$ disease $(n=9)$ exhibited $\beta$-migrating VLDL $\left(\mathrm{S}_{\mathfrak{f}}>20\right)$ which were abnormally rich in cholesterol $(1,2)$. Those with "mixed lipemia" $(n=10)$ displayed the electrophoretic pattern of type V hy-

\footnotetext{
${ }^{1}$ Throughout this paper the term chylomicron will be restricted to designate "primary particles" (3), which have an $\mathrm{S}_{\mathbf{r}}>400$, are composed to a large extent of dietary triglyceride, and collect as a flocculate at the top of a $3 \%$ polyvinylpyrollidone (PVP) column after mixing plasma with $5 \% \mathrm{PVP} / 10 \% \mathrm{NaCl}$ and overnight incubation at $37^{\circ} \mathrm{C}$ (4). Thus "secondary particles" (3), also largely of exogenous origin, and "hyperlipemic particles" (5), of endogenous origin, both of which are $S_{t}>400$, have been excluded in the present study of chylomicron composition in broad- $\beta$ disease and other forms of hyperlipoproteinemia.
} 
TABLE I

Triglyceride and Cholesterol Content of Chylomicrons Persisting after an Overnight Fast: Broad- $\beta$ Disease vs. Mixed Lipemia

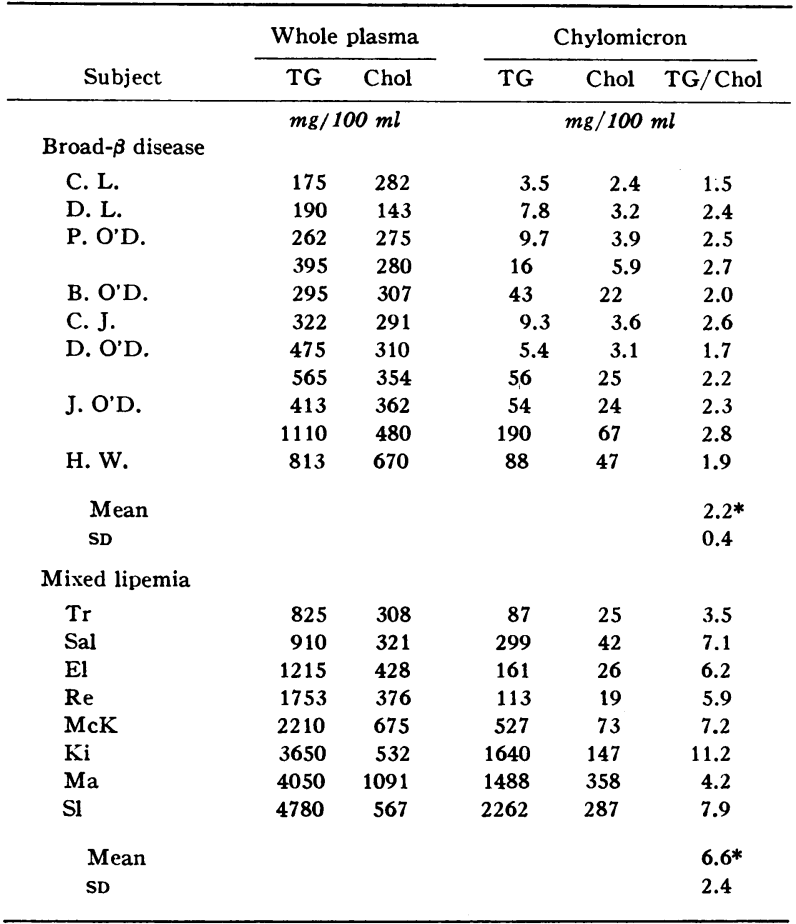

* Group mean calculated from average of ratios where two determinations were made on different occasions in the same subject. Difference between means highly significant $(P<0.001)$.

perlipoproteinemia (1) and had circulating chylomicrons * after an overnight fast. The persistence of these chylomicrons, largely composed of dietary TG, was presumptive evidence of an element of impaired TG removal. In addition, studies of TG turnover (6) demonstrated both a high rate of endogenous TG turnover and high levels of VLDL. Hence the term "mixed lipemia" (exogenous plus endogenous) has been applied. The subject with endogenous lipemia had a type IV electrophoretic pattern (1), a high rate of TG turnover, and increased VLDL levels but no chylomicrons in the fasting state. Those with familial hypercholesterolemia $(n=3)$ exhibited the characteristic hyperbetalipoproteinemia (type II pattern [1]) and tendinous xanthomas.
Laboratory determinations. Blood samples were collected in EDTA, $1 \mathrm{mg} / \mathrm{ml}, 12-14 \mathrm{hr}$ after the last meal while the subjects were consuming weight-maintaining diets. Plasma was separated promptly and remained at room temperature until extraction or incubation. Analyses of TG (7) and cholesterol (8) were made from extracts in chloroform: methanol $(2: 1, \mathrm{v}: \mathrm{v})$.

All studies of chylomicron composition were performed on primary particles isolated by PVP flocculation (4). The flocculated chylomicrons were aspirated from the top $5 \mathrm{~mm}$ of a $78 \mathrm{~mm}$ tube after slicing. Incubations with PVP were performed with several different volumes of each plasma sample and the tubes sliced only if a clear zone was visible between the flocculate and the subjacent "hyperlipemic particles" (5). The isolated chylomicrons were analyzed for TG and cholesterol after removal of PVP (4).

Oral fat load studies. Oral fat loads, $2 \mathrm{~g} / \mathrm{kg}$, were administered either as whipping cream or as corn or linseed oil blended with skim milk. Plasma was collected for chylomicron analysis at varying intervals during the 4-24 $\mathrm{hr}$ thereafter. No other food was ingested during the course of the study. The subject given linseed oil (55.2\% linolenic acid) was a $24 \mathrm{yr}$ old male with broad- $\beta$ disease (D. L., Table I) who had been maintained on an isocaloric formula diet $(40 \%$ fat, $45 \%$ carbohydrate, $15 \%$ protein) for 3 wk before his fat load. The triglyceride fatty acid composition of his primary particles was determined at 4-hr intervals for the duration of chylomicronemia $(20 \mathrm{hr})$ by methods previously described (9), which included thin-layer silicic acid chromatography for separation of triglycerides from chloroform-methanol lipid extracts and gas-liquid chromatography for analysis of the triglyceride fatty acid methyl esters.

In vitro incubation studies. To assess in vitro changes in chylomicron composition, whole plasma samples collected from three subjects at the peak of lipemia after oral fat loading were incubated with shaking at $37^{\circ} \mathrm{C}$ for $16-24 \mathrm{hr}$ before isolation of chylomicrons (Table II). In other studies (Table III) plasmas drawn $6 \mathrm{hr}$ after an oral fat load were first fractionated into $S_{t}>400$ and $S_{t}<400$ lipoproteins and then incubated as follows: $S_{t}>400$ lipoproteins from a broad- $\beta$ subject with $S_{t}<400$ lipoproteins from a mixed lipemic, and $S_{t}>400$ lipoproteins from the mixed lipemic with $S_{\mathbf{f}}<400$ lipoproteins from the broad- $\beta$ subject. Lipoprotein fractions for incubation were prepared as follows : plasma was centrifuged for $7.95 \times 10^{\circ} \mathrm{g}$-min in a $41 \mathrm{Ti}$ swinging bucket rotor; the $\mathrm{S}_{\boldsymbol{t}}<400$ lipoproteins in the lower $11.5 \mathrm{ml}$ were drained after tube puncture; the $S_{f}>400$ supernatant was recentrifuged in $d 1.006$ saline, collected by aspiration, and suspended in $6 \mathrm{ml}$ saline. $1 \mathrm{ml}$ of this suspension was then incubated with shaking at $37^{\circ} \mathrm{C}$

TABLE II

Change in Lipid Composition of Chylomicrons in Whole Plasma Obtained 4-6 hr after an Oral Fat Load during Incubation In Vitro $\left(16-24 \mathrm{hr}, 37^{\circ} \mathrm{C}\right)$

\begin{tabular}{|c|c|c|c|c|c|c|c|c|c|}
\hline \multirow[b]{3}{*}{ Subject } & \multirow{3}{*}{$\begin{array}{l}\text { Type of hyper- } \\
\text { lipoproteinemia }\end{array}$} & & & \multicolumn{6}{|c|}{ Chylomicrons } \\
\hline & & \multicolumn{2}{|c|}{ Whole plasma } & \multicolumn{3}{|c|}{ Preincubation } & \multicolumn{3}{|c|}{ Postincubation } \\
\hline & & TG & Chol & TG & Chol & TG/Chol & TG & Chol & TG/Chol \\
\hline & & \multicolumn{2}{|c|}{$m g / 100 \mathrm{ml}$} & \multicolumn{3}{|c|}{$m g / 100 \mathrm{ml}$} & \multicolumn{3}{|c|}{$m \mathrm{~m} / 100 \mathrm{ml}$} \\
\hline H. W. & Broad $-\beta$ disease & 1354 & 430 & 575 & 55 & 10.5 & 450 & 62 & 7.3 \\
\hline B. O'D. & Broad $-\beta$ disease & 695 & 345 & 196 & 18 & 10.9 & 123 & 19 & 6.4 \\
\hline San & Mixed lipemia & 2060 & 312 & 836 & 63 & 13.3 & 530 & 48 & 11.3 \\
\hline
\end{tabular}


TABLE III

Change in Lipid Composition of Chylomicrons in $S_{f}>400$ Lipoproteins Obtained $6 \mathrm{hr}$ after an Oral Fat Load during Incubation In Vitro $\left(16 \mathrm{hr}, 37^{\circ} \mathrm{C}\right)$ with Plasma Containing No Chylomicrons

\begin{tabular}{|c|c|c|c|c|c|c|c|}
\hline \multirow[b]{2}{*}{ Experiment } & \multicolumn{3}{|c|}{ Source of chylomicrons } & \multicolumn{4}{|c|}{ Source of chylomicron-free plasma } \\
\hline & Subject & $\begin{array}{l}\text { Type of hyper- } \\
\text { lipoproteinemia }\end{array}$ & $\begin{array}{c}\text { Preincubation } \\
\text { chylomicron } \\
\text { TG/Chol }\end{array}$ & Subject & $\begin{array}{l}\text { Type of hyper- } \\
\text { lipoproteinemia }\end{array}$ & $\begin{array}{c}\text { Cholesterol } \\
\text { concentration }\end{array}$ & $\begin{array}{c}\text { Postincubation } \\
\text { chylomicron } \\
\text { TG/Chol* }\end{array}$ \\
\hline & & & & & & $m g / 100 m l$ & \\
\hline A & San & Mixed & 13.3 & H. W. & Broad- $\beta$ & 360 & 6.9 \\
\hline B & $"$ & $"$ & $"$ & S. H. & $\begin{array}{l}\text { Familial } \\
\text { hypercholesterolemia }\end{array}$ & 446 & 6.7 \\
\hline II A & H. W. & Broad $-\beta$ & 10.5 & San & Mixed & 182 & 11.3 \\
\hline B & " & " & $"$ & S. H. & $\begin{array}{l}\text { Familial } \\
\text { hypercholesterolemia }\end{array}$ & 446 & 9.0 \\
\hline
\end{tabular}

* Incubations performed in duplicate; coefficient of variation between duplicates $=10 \%$.

with $2 \mathrm{ml}$ of the appropriate $\mathrm{S}_{\mathrm{f}}<400$ infranatant. After $16 \mathrm{hr}$ primary particles were collected for lipid analysis. Similar incubations of the $S_{f}>400$ fraction from each subject were performed with fasting whole plasma from a subject with familial hypercholesterolemia (TG $52 \mathrm{mg} / 100 \mathrm{ml}$, cholesterol $446 \mathrm{mg} / 100 \mathrm{ml}$, origin-staining material absent by electrophoresis).

\section{RESULTS}

Chylomicrons persisting after an overnight fast. The persistence of chylomicrons ${ }^{1}$ after an overnight fast was not anticipated in early studies of broad- $\beta$ disease but has since proven a common finding. Fasting chylomicrons have been detected at plasma TG concentrations as low as $175 \mathrm{mg} / \mathrm{ml}$ and have been observed regularly at levels of $300-500 \mathrm{mg} / 100 \mathrm{ml}$.

The composition of these fasting chylomicrons was distinctive: they contained consistently larger proportions of cholesterol and less TG than fasting chylomicrons from subjects with mixed lipemia (Table I), the other group studied in which chylomicrons were observed after an overnight fast. Thus, the mean ratio of chylomicron TG/cholesterol in the subjects with broad- $\beta$ disease was depressed significantly below the mean ratio among the mixed lipemics $(P<0.001)$, with no overlap between the two groups. Although the quantity of chylomicron lipid in subjects with broad- $\beta$ disease tended to be less than in subjects with mixed lipemia, in several of the latter ( $\mathrm{Tr}, \mathrm{El}$, and $\mathrm{Re}$ ), even small quantities of chylomicrons retained their TG-rich composition. Thus, a TG/cholesterol ratio of $<3.0$ in fasting chylomicrons appeared to be characteristic of broad- $\beta$ disease.

In spite of their abnormal composition, the fasting chylomicrons observed in subjects with broad- $\beta$ disease resembled primary particles previously characterized in normal subjects (9) and subjects with other forms of hyperlipemia (5) in the following respects :

(a) They were of $\mathrm{S}_{\mathrm{t}}>400: \mathrm{S}_{\mathrm{f}}>400$ plasma produced a flocculate at the top of the PVP column; $S_{t}<400$ plasma did not (subject H. W., Table I) ;

(b) Primary particles disappeared rapidly on a fatfree ( $85 \%$ carbohydrate) diet despite an increase in plasma TG levels and the persistence of $\beta$-migrating

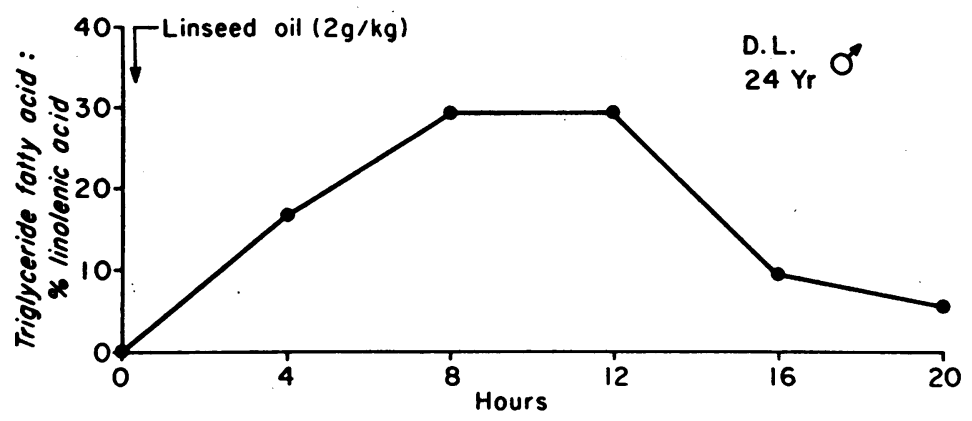

FIGURE 1 Chylomicron lipid composition during alimentary lipemia. The proportion of linolenic acid in primary particles isolated from a subject with broad- $\beta$ disease before and after an oral fat load (linseed oil, $2 \mathrm{~g} / \mathrm{kg}$ ). 
VLDL of abnormal, cholesterol-rich composition (subject H. W.) ;

(c) The TG fatty acid pattern of primary particles isolated both early and late after an oral fat load in subject D. L. (Fig. 1) reflected the presence of the oil ingested (admixed to an increasing extent with circulating and newly synthesized endogenous TG [3]).

Chylomicrons after an oral fat load. To determine the origin of the abnormal composition of fasting chylomicrons in broad- $\beta$ disease, the dynamics of chylomicron lipid composition in response to exogenous $\mathrm{TG}$ were assessed by giving oral fat loads, $2 \mathrm{~g} / \mathrm{kg}$, to five subjects with broad- $\beta$ disease and, for comparison, two with mixed lipemia, one with endogenous lipemia, and two with familial hypercholesterolemia (Fig. 2). In each subject with broad- $\beta$ disease the $T G /$ cholesterol ratio of primary particles at peak chylomicronemia was markedly higher than in the fasting state and was equivalent to the ratios observed in the other forms of hyperlipoproteinemia. As the alimentary lipemia subsided, however, the $\mathrm{TG} /$ cholesterol ratio in chylomicrons remaining in subjects with broad- $\beta$ disease declined to the level observed in the fasting state but was not appreciably below the ratio recorded in subjects with familial hypercholesterolemia at a similar point in time. In subjects with either mixed or endogenous lipemia, on the other hand, the chylomicron $\mathrm{TG}$ /cholesterol ratio remained high despite a moderate reduction from the level observed at peak chylomicronemia.

Changes in chylomicron composition during incubation in vitro. In order to determine whether the observed lipid composition of fasting chylomicrons in broad- $\beta$ disease might result from excessive activity of cholesterol transfer mechanisms, chylomicrons from plasma collected at the peak of alimentary lipemia from two subjects with broad- $\beta$ disease and one with mixed lipemia were analyzed both before and after incubation at $37^{\circ} \mathrm{C}$

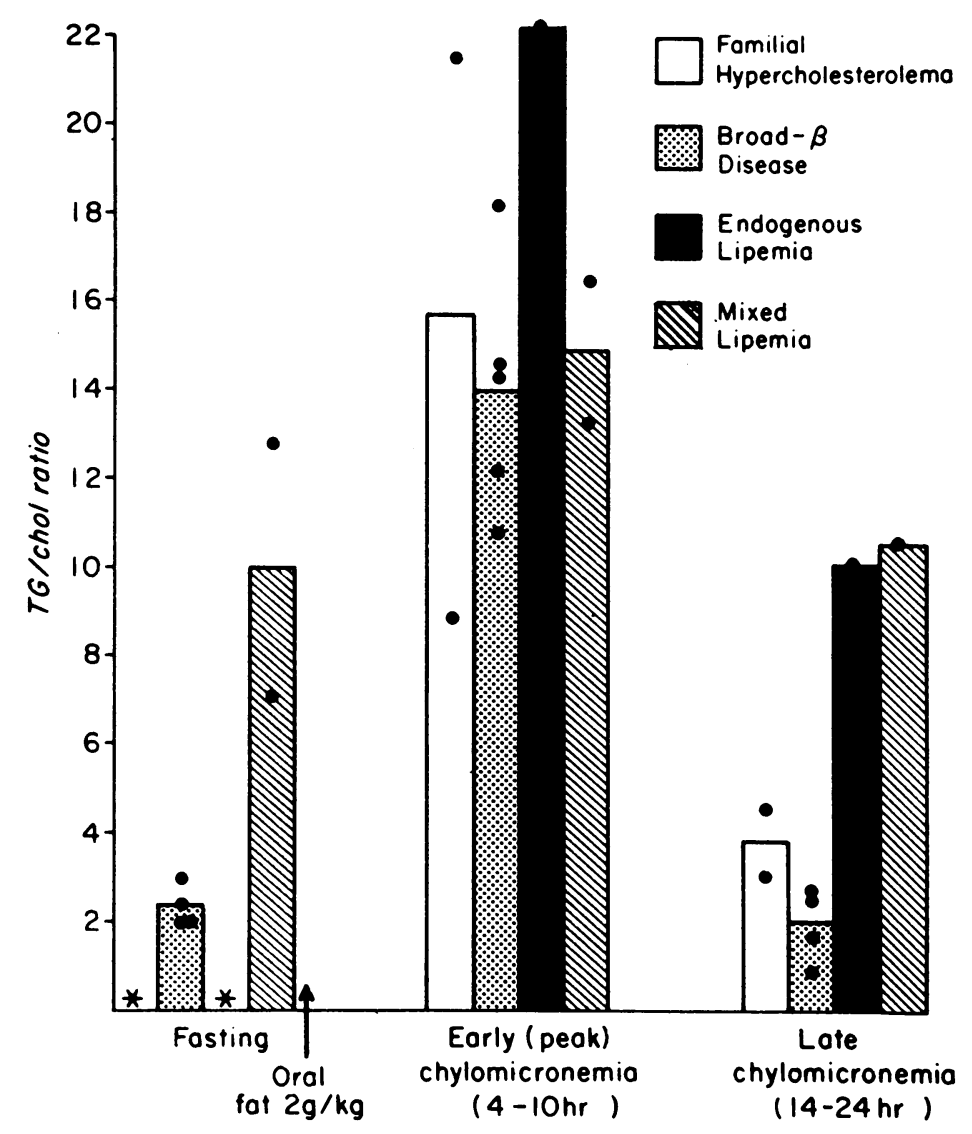

FIgURE 2 Dietary triglyceride in chylomicrons in broad- $\beta$ disease. Ratios of chylomicron TG/cholesterol before and after oral fat loads, 2 $\mathrm{g} / \mathrm{kg}$, in subjects with hyperlipoproteinemia. The solid circles represent values obtained in individual subjects, and the bars show mean values. * Subjects with familial hypercholesterolemia and endogenous lipemia had no chylomicrons in the fasting state. 
in vitro (Table II). In both types of lipemia the TG/ cholesterol ratio declined during incubation but did so to a lesser degree in the mixed lipemic.

Factors influencing changes in chylomicron composition were explored before and after incubation of $\mathrm{S}_{\mathbf{r}}>$ 400 lipoproteins collected after an oral fat load from a subject with mixed lipemia with particle-free plasma $\left(S_{\mathbf{t}}<400\right)$ from a broad- $\beta$ subject (and conversely) or with whole fasting plasma from a subject with familial hypercholesterolemia (Table III). In these studies chylomicrons from the subject with mixed lipemia showed a relative accumulation of cholesterol to the same (or greater) degree as did those from the broad- $\beta$ subject when both were incubated with plasma from the familial hypercholesterolemic subject (experiment I B vs. II B). Thus excessive avidity for cholesterol uptake was not evident in broad- $\beta$ chylomicrons. Furthermore, chylomicrons from the subject with mixed lipemia became no more cholesterol-rich during incubation with particle-free plasma from the broad- $\boldsymbol{\beta}$ subject than during incubation with plasma from the familial hypercholesterolemic subject (experiment I A vs. I B). Therefore, no unique influence on chylomicron composition was evident in $\mathrm{S}_{\mathfrak{t}}<$ 400 lipoproteins in broad- $\beta$ plasma.

Instead, in all experiments the composition of chylomicrons following incubation appeared to reflect the relative concentration of cholesterol in the nonparticulate lipoproteins in the medium: the TG/cholesterol ratio declined further in the presence of cholesterolrich $S_{\mathrm{r}}<400$ plasma, whether from the subject with broad- $\beta$ disease or with familial hypercholesterolemia, than in the presence of cholesterol-poor $\mathrm{S}_{\mathrm{f}}<400$ plasma from the mixed lipemic.

\section{DISCUSSION}

The persistence of circulating chylomicrons ${ }^{1}$ after an overnight fast has not previously been stressed as a characteristic of broad- $\beta$ disease. Nevertheless, this phenomenon has been observed in a large proportion of subjects with broad- $\boldsymbol{\beta}$ disease in this laboratory, both by PVP flocculation and agarose electrophoresis, and has proven an early clue to this difficult diagnosis in several cases. Of particular note has'been the occurrence of fasting chylomicronemia at relatively low plasma TG levels, $300-500 \mathrm{mg} / 100 \mathrm{ml}$, the range usually observed in subjects with endogenous lipemia (in whom fasting chylomicronemia is not detectable), and well below that ordinarily observed in subjects with mixed lipemia, whose plasma TG concentrations usually exceed $750 \mathrm{mg} /$ $100 \mathrm{ml}$. Furthermore, definitive separation of subjects with broad- $\beta$ disease from those with mixed lipemia may be made by lipid analysis of fasting chylomicrons and calculation of the TG/cholesterol ratio.

The low ratio observed in broad- $\beta$ disease indicates that fasting chylomicrons display the abnormal lipid composition (relatively rich in cholesterol and poor in TG) present within the entire VLDL spectrum in this disorder (7). These chylomicrons share the property of flocculation and flotation in 3\% PVP columns characteristic of particles of dietary origin in normal subjects (9) and those with other forms of hyperlipemia (5); therefore, studies of chylomicron composition during assimilation of an oral fat load were undertaken to determine the source of the abnormal composition of chylomicrons which persist after an overnight fast in subjects with broad $\boldsymbol{\beta}$ disease.

These experiments, together with the results of in vitro studies, suggest that chylomicrons are normal when secreted by the intestine in broad- $\beta$ disease but become rich in cholesterol by virtue of exaggerated lipid exchanges $(10,11)$, in addition to lipolysis, which take place during prolonged circulation in a cholesterol-rich medium. This conclusion is supported by the following evidence: $(a)$ chylomicrons from broad- $\beta$ subjects were of normal composition at peak chylomicronemia following an oral fat load $(12) ;(b)$ as alimentary lipemia subsided, remaining chylomicrons were rich in cholesterol; (c) chylomicrons from subjects with familial hypercholesterolemia showed a similar cholesterol enrichment late after a fat load; and $(d)$ chylomicrons from a subject with mixed lipemia became as rich in cholesterol as did broad- $\beta$ chylomicrons during incubation with hypercholesterolemic plasma.

Finally, since enrichment of chylomicrons with cholesterol was observed in forms of hyperlipoproteinemia other than broad- $\beta$ disease, these studies are consistent with the hypothesis that the abnormal composition of fasting chylomicrons in broad- $\beta$ disease may arise through processes which are not unique to this disorder.

\section{ACKNOWLEDGMENTS}

We wish to acknowledge the excellent technical assistance of Eileen Severns, Y. L. Lee, and Karen Tilbury.

This work was supported by U. S. Public Health Training Grant AM-1000, Research Project Grant AM-06670, and Clinical Research Center Grants 5 M01-R37 (University Hospital) and FR-133 (Harborview Medical Center). Dr. Porte and Dr. Bierman received Career Development Awards 5-K4-AM-8865 and AM-28167, respectively.

\section{REFERENCES}

1. Fredrickson, D. S., R. I. Levy, and R. S. Lees. 1967. Fat transport in lipoproteins-an integrated approach to mechanisms and disorders. N. Engl. J. Med. 276: 34, 94, 148, 215, 273.

2. Hazzard, W. R., F. T. Lindgren, and E. L. Bierman. 1970. Very low density lipoprotein subfractions in a subject with broad- $\beta$ disease (Type III hyperlipoproteinemia) and a subject with endogenous lipemia (Type IV) : chemical composition and electrophoretic mobility. Biochim. Biophys. Acta. 202: 517. 
3. Zilversmit, D. B. 1969. Chylomicrons. In Structural and Functional Aspects of Lipoproteins in Living Systems. E. Tria and A. M. Scanu, editors. Academic Press Inc., New York. C1: 329.

4. O'Hara, D. D., D. Porte, Jr., and R. H. Williams. 1966. Use of constant composition polyvinylpyrrolidone columns to study the interaction of fat particles with plasma. J. Lipid Res. 7 : 264.

5. Bierman, E. L., D. Porte, Jr., D. D. O'Hara, M. Schwartz, and F. C. Wood, Jr. 1965. Characterization of fat particles in plasma of hyperlipemic subjects maintained on fat-free high-carbohydrate diets. J. Clin. Invest. 44: 261 .

6. Porte, D., Jr., and E. L. Bierman. 1969. The effect of heparin infusion on plasma triglyceride in vivo and in vitro with a method for calculating triglyceride turnover. J. Lab. Clin. Med. 73: 631.

7. Bierman, E. L., and J. T. Hamlin III. 1961. The hyper- lipemic effect of a low-fat, high-carbohydrate diet in diabetic subjects. Diabetes. 10: 432 .

8. Searcy, R. L., L. M. Bergquist, and R. C. Jung. 1960. Rapid ultramicro estimation of serum total cholesterol. J. Lipid Res. 1: 349.

9. Bierman, E. L., E. Gordis, and J. T. Hamlin, III. 1962. Heterogeneity of fat particles in plasma during alimentary lipemia. J. Clin. Invest. 41: 2254.

10. Nichols, A. V., and L. Smith. 1965. Effect of very low density lipoproteins on lipid transfer in incubated serum. J. Lipid Res. 6: 206.

11. Akanuma, Y., and J. A. Glomset. 1968. In vitro incorporation of cholesterol- ${ }^{14} \mathrm{C}$ into very low density lipoprotein cholesterol esters. J. Lipid Res. 9: 620.

12. Porte, D., Jr., D. D. O'Hara, and R. H. Williams. 1966. Lipid composition of fat particles from normal man and patients with idiopathic hypertriglyceridemia. J. Lipid Res. 7 : 368. 